\title{
Éditorial du comité de direction. Quoi de neuf après dix numéros?
}

Karine Ginisty, Amandine Spire et Jeanne Vivet

\section{OpenEdition}

\section{Journals}

Édition électronique

URL : https://journals.openedition.org/cdg/1683

DOI : $10.4000 /$ cdg. 1683

ISSN : 2107-7266

Éditeur

UMR 245 - CESSMA

\section{Référence électronique}

Karine Ginisty, Amandine Spire et Jeanne Vivet, «Éditorial du comité de direction. Quoi de neuf après dix numéros ? », Carnets de géographes [En ligne], 11 | 2018, mis en ligne le 15 novembre 2018, consulté le 22 mai 2021. URL : http://journals.openedition.org/cdg/1683 ; DOI : https://doi.org/ $10.4000 /$ cdg. 1683

Ce document a été généré automatiquement le 22 mai 2021.

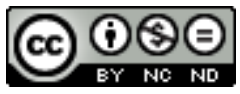

La revue Carnets de géographes est mise à disposition selon les termes de la Licence Creative Commons Attribution - Pas d'Utilisation Commerciale - Pas de Modification 4.0 International. 


\title{
Éditorial du comité de direction. Quoi de neuf après dix numéros?
}

\author{
Karine Ginisty, Amandine Spire et Jeanne Vivet
}

1 Pour le onzième numéro de la revue, l'équipe des Carnets de géographes a fait une pause dans l'élaboration de numéros spéciaux ciblant des domaines de recherches spécifiques et a lancé un appel à contributions pour un numéro non thématique (Varia).

2 La préparation de ce 11ème numéro a également été un moment de réflexion sur la trajectoire de la revue depuis sa création et sur son positionnement dans le paysage éditorial et dans le champ de la géographie. Le comité de direction a souhaité ainsi revenir sur la création, les expérimentations et le positionnement de la revue à travers le texte introduisant le 11ème numéro.

3 Ce texte se nourrit des nombreux échanges qui ont pris place lors des comités de rédaction.

4 Nous remercions chaleureusement Jean Estebanez, Marianne Morange et Margot Beauchamps pour l'entretien qu'ils nous ont accordé le 13 septembre 2018 et qui nous a aidé à formaliser le texte présenté ici.

5 Un processus de renouvellement des comités a caractérisé l'année 2018 afin que de nouvelles pages des Carnets puissent s'écrire. Le prochain numéro viendra préciser les inflexions en cours.

\section{Tout n'est pas neuf ...}

6 Depuis sa création, la revue a été pensée comme un espace éditorial pouvant participer de la consolidation d'une géographie ouverte aux autres sciences sociales, et ce afin de permettre son renouvellement. Donner à voir des démarches innovantes ou émergentes représente tout un programme. Concrètement, les dix premiers numéros de la revue ont tout d'abord modestement contribué à un travail d'unification de travaux menés de façon dispersée dans un champ spécifique, comme par exemple le numéro portant sur les faits religieux, ou celui sur les espaces de l'entre-deux. Par 
ailleurs, certains numéros ont participé à la discussion de thématiques déjà bien constituées dans la sphère anglophone (les approches critiques, les enfants, les animaux, les émotions) mais encore peu mobilisée dans la géographie francophone. Plus qu'à innover, le projet a finalement peut-être davantage consisté à essayer de transformer les façons de voir des objets, d'adopter des démarches, des champs théoriques auprès d'un lectorat majoritairement géographe. Les Carnets ont ainsi tenté de discuter d'objets non identifiés a priori comme relevant de la discipline géographique : l'animal, le numérique, en les rendant plus légitimes au sein de la communauté des géographes.

\section{Un renouvellement en lien avec d'autres sciences sociales et humaines}

7 Le renouvellement passait par l'ouverture aux autres sciences sociales et humaines. Le projet s'adressait initialement aux géographes dans l'optique de nourrir leur positionnement par la lecture de corpus théoriques, de travaux, n'appartenant pas directement à la géographie. Les appels à contributions ont ainsi à chaque fois été l'occasion de constituer une bibliographie qui allait au-delà des travaux de géographie afin de dépasser certaines approches déjà bien balisées dans la discipline. L'emprunt à d'autres disciplines contribue à alimenter, en creux, les discussions sur ce qu'est la géographie, sur ses fondements et ses possibles (souhaitables?) évolutions. Chaque numéro a tenté de mettre en regard des approches disciplinaires différentes des objets convoqués. Les propositions d'articles reçues ainsi que les papiers publiés après évaluation reflètent une ouverture en terme d'appartenance disciplinaire des auteurs et des relecteurs. On dénombre 27 publications d'auteurs relevant d'autres disciplines que la géographie, majoritairement la sociologie mais aussi l'anthropologie, l'urbanisme, les sciences de l'éducation...

8 Ceci étant, toutes les géographies ne sont pas représentées dans les Carnets de géographes. Les approches sociales et politiques dominent très largement en raison des orientations initiales du comité de direction et de rédaction. La géographie physique est par exemple absente. Les questions environnementales, qui occupent un pan important des débats géographiques contemporains, n'ont pas non plus été développées à ce jour.

\section{Promouvoir une certaine liberté d'écriture}

9 Les Carnets proposaient d'ouvrir leurs pages à des modalités d'écriture moins conventionnelles. Il ne s'agissait pas d'une injonction mais d'une possible invitation, en considérant, à tort certainement, qu'écrire autrement permettrait de sortir de certaines normes académiques pouvant être limitantes. Après dix numéros, extrêmement peu d'auteurs se sont saisis de cette possibilité, préférant s'en tenir aux codes de communication scientifique classique. Ces normes ne sont pas uniquement des carcans. Elles facilitent évidemment la réception tout en participant de la validité de la démarche scientifique. Bien qu'ouverts à d'autres modalités d'écriture, les Carnets de géographes restent une revue scientifique avec des procédures de publication classiques et non un blog ou un ouvrage en ligne. 


\section{Les Carnets de terrain : consolider et donner à voir les méthodes d'enquêtes de terrain.}

10 Si les enquêtes de terrain sont bien balisées et travaillées dans certaines disciplines (anthropologie, sociologie), la géographie s'est longtemps caractérisée à la fois par l'importance du terrain comme fondement de la démarche scientifique et son silence sur les méthodes mises en œuvre. Effectuées de manière très inductive et transmises de façon individualisées, les pratiques de terrain ont longtemps été mises à l'arrière-plan, reléguées au mieux dans des annexes de thèse de doctorat. La revue a été créée à la fin des années 2000 au moment de la montée des approches qualitatives, de leur formalisation, et de leur inscription dans des cursus de géographie.

11 La création d'une rubrique spécifiquement dédiée aux pratiques de terrain a été une forme d'innovation dans l'édition scientifique géographique. Il s'agissait de mettre en récit les coulisses de la recherche en train de se produire, dans une démarche réflexive et critique. La façon de mener l'enquête de terrain pouvait alors constituer un résultat en soi tout aussi intéressant que le résultat final de la recherche. La formation de cette rubrique a coïncidé avec la formalisation des techniques qualitatives en géographie. Cet effort de légitimation du qualitatif s'est peut-être opéré au détriment des approches quantitatives peu représentées dans les Carnets, et qui y ont pourtant tout autant leur place. Idéalement, le croisement des approches qualitatives et quantitatives pourrait s'exposer et se discuter dans cette rubrique.

12 Ces Carnets de terrain ont par ailleurs une certaine vertu pédagogique dans la mesure où ils mettent en scène les étapes de la production scientifique, en la désacralisant. Faire de la recherche n'apparaît plus comme une boîte noire mais comme une suite de pratiques conditionnées par la maîtrise de certains outils et de paramètres non forcément fixés a priori. La prise en compte de la présence et de la subjectivité du chercheur dans la conduite et l'analyse de l'enquête, déjà bien étudiée par ailleurs, prenait ainsi plus nettement place en géographie. Les retours des lecteurs nous ont montré que les articles publiés dans cette rubrique étaient fréquemment mobilisés dans le cadre d'enseignements de licence et de Master. Plus que fournir des recettes toutes prêtes pour mener une enquête, ces textes proposent de multiples récits et retours d'expériences (44 articles publiés dans les 10 premiers numéros) propices à la réflexivité et au cheminement personnel des étudiants comme des enseignants.

La formalisation de cette étape de la démarche géographie pose toutefois des questions. Exposer les coulisses de la recherche peut devenir un attendu, une injonction, appelant à la mise en récit de soi, sous un certain angle, que l'on voudrait transparent et authentique. Cela demande une certaine disposition inégalement partagée qui produit des effets de légitimation et de normation, afin de présenter ce qui deviendrait la bonne démarche méthodologique.

\section{La question du positionnement politique de la revue vis-à-vis des pratiques de classement}

14 En 2008, l'AERES a fait un classement des revues scientifiques qui a entraîné beaucoup d'émoi et de discussions. La revue s'est initialement constitué comme un espace 
éditorial refusant de participer à l'homologation de la production scientifique dans une position de résistance aux critères alors imposés (notamment la question de l'impact factor et de l'internationalisation des comités et du lectorat). Depuis dix ans, l'AERES, devenue HCERES, n'a pas reconduit son opération de classement même si cela reste à son agenda. La revue a suscité et encouragé particulièrement la diffusion de travaux de jeunes chercheurs pour qui les conditions de reconnaissance et d'évaluation passent de plus en plus par des publications dans des revues reconnues institutionnellement. Cela pourrait poser à nouveau le débat sur l'intégration des critères de l'HCERES dans le fonctionnement des Carnets de géographes. Ceci étant, aller à l'encontre de l'uniformisation des pratiques de recherche pour pouvoir laisser place à des démarches marginales, hésitantes, en cours de maturation, constitue toujours un point central du projet de la revue.

INDEX

Thèmes : Carnets de débats 